showed values consistent with the OPSIS measurements. The measurement methods for nitrogen dioxide before OPSIS did not give 24 hour levels and cannot be compared. At the time of the study the OPSIS system was the only one measuring ozone in the area. This criticism raises a more important point which we refer to in our paper but which deserves emphasis. In most epidemiological studies pollution measurements are made from a static monitoring site(s). Whether this is at a roadside or on a roof, it can only give an approximate estimate of the exposure of subjects who may spend time some distance away and who will spend much of their time indoors. Our study, previous Birmingham studies, and most similar work will suffer from this inaccuracy until individual monitors measuring multiple exposures are available.

We agree completely with the correspondents' comments about causality. A study such as ours can only demonstrate associations as stated in our paper, but we do not agree that we have been notably less cautious than the Birmingham group.

We are surprised by the remarks concerning nitrogen dioxide challenge. We mention challenge tests in our introduction but make it clear that, although changes can be identified in such tests, the circumstances in which they are performed are highly artificial. Nowhere do we imply that effects of nitrogen dioxide are seen in the laboratory at ambient levels.

B G HIGGINS
A WOODCOCK
Lung Function Unit,
North West Lung Centre,
Wythenshawe Hospital,
Manchester M23 9LT,
UK

1 Whittemore AS, Korn EL. Asthma and air pollution in the Los Angeles area. Am $\mathcal{F}$ Public Health 1980;70:687-97.

2 Bland JM, Altman DG. Calculating correlation coefficients with repeated observations. Part 1. Correlation within subjects. $B M \mathcal{F} 1995 ; 310$ 446.

3 Rogan JC, Keselman HJ; Mendoza JL. Analysis of repeated measurements. $\mathrm{Br} \mathcal{F}$ Math Stat Psychol 1979;32:269-86.

4 Walters SM, Griffiths RM, Ayres JG. Temporal association between hospital admissions for admissions for asthma in Birmingham and ambient levels of sulphur dioxide and smoke. Thorax 1994;49:133-40.

\section{Fatal chickenpox pneumonia in asthma}

The interesting case report of Drs Gatnash and Connolly (April 1995;50:422-3) reminds us that chickenpox may be fatal in an immunosuppressed patient. I agree with the authors' recommendations for prevention, but would add one thing. At-risk patients exposed to chickenpox or herpes zoster should seek urgent medical attention for antibody screening and, if antibody negative, should receive passive immunisation with varicella zoster immunoglobulin (VSIG). ${ }^{12}$

CRAIG SKINNER

Department of Respiratory Medicine, Birmingham Heartlands Hospital, Bordesley Green East, Birmingham B9 $5 S S$

1 Department of Health. Immunization against infectious disease. London: HMSO, 1992.

2 Severe chickenpox associated with systemic corticosteroids. In: Current problems in pharmaco vigilance. Committee on Safety of Medicines and the Medicines Control Agency, 1994;20 $1-2$.

\section{BOOK NOTICES}

A Colour Atlas of Respiratory Diseases. 2nd edition. D Geraint James and Peter R Studdy. (Pp 366; £71.00). London: Wolfe Publishing, 1993. 0723416958.

This is the second edition of a very successful atlas. The first edition, published in 1981, lacked several important recent developments, particularly CT scanning and respiratory aspects of HIV and the immunocompromised patient which are now well covered. There are also new sections on sleep apnoea, MRI, parasitic disease and pulmonary vascular disease. The book appeals to a wide audience, including the more enthusiastic medical student, MRCP candidates, and respiratory nurse specialists. It won't harm senior thoracic physicians either!

The authors modestly refer the reader to other textbooks for details but the captions and brief texts accompanying the figures do, nevertheless, provide quite a lot of information. This is very adequate for the depth of knowledge that the respiratory nurse might want to acquire and a useful review for the MRCP candidate. There are many useful classifications and tables.

There is a major new contribution from Basil Strickland to the radiology in the atlas, particularly with the inclusion of CT images in all the conditions where this is an important investigation. Interpretation of the plain chest radiograph is also dealt with very well. This section is particularly commended to junior doctors who frequently seem to have difficulty in mastering the basic principles involved in distinguishing between major features such as collapse and consolidation - an unhealthy situation both for the patient and success in examinations!

This is a wonderful book to just browse through and represents a unique collection of slides from the authors and some $70 \mathrm{col}-$ leagues. It is a very good example of a picture being worth a thousand words and an excellent way to both learn and revise. It is strongly recommended for a wide readership. My only criticism is that the outside cover is not strong enough for the heavy use to which the atlas will be subjected. - MRH

Tuberculosis - A Clinical Handbook. Larry I Lutwick. (Pp 378; f25.00). London: Chapman \& Hall, 1994. 0412607409.

This book, dealing with many aspects of tuberculosis, has a multi-contributor authorship which is entirely from the United States, mainly from New York. There are chapters on the history and epidemiology of tuberculosis, pulmonary and non-pulmonary disease in adults, paediatric aspects, and microbiology. Because of the all American authorship there is a major bias to the USA in management, ethics, and references quoted, which is both a strength and a weakness. The sections on the epidemiology and clinical aspects of multiple drug resistant tuberculosis are up to date, well referenced, and give a good overview, with potential regimens in both HIV negative and HIV positive patients. Some of these regimens, however, are speculative and not evidentially based.

The section on ethical and legal aspects of tuberculosis control is virtually only applicable to the USA; that on infection control concentrates significantly on chemical agents, personal respiratory protection, and ventilation systems to levels which are not felt necessary on this side of the Atlantic except under exceptional circumstances, and are irrelevant for developing countries. For a UK readership there are significant gaps. Under tuberculin testing the Heaf test is barely mentioned, BCG vaccination and its pros and cons are only briefly covered, and the benefits of BCG vaccination for health care workers in particular are not given a balanced assessment. Non-tuberculous mycobacteria are covered individually in a separate chapter. This section does not explain the general principle that individual drug sensitivities are to be ignored, that clinical combinations often work even though the organisms are resistant in vitro, or that drug sensitivity tests using clinical combinations often show different results.

The book is cheaper than a number of other books on the topic, but does not add much for the UK reader which is not already available in other texts. The sections on multiple drug resistant tuberculosis will be of use in specialised situations and may be usefully consulted on such occasions. - LPO

\section{NOTICES}

\section{RCN Tuberculosis Visitors}

\section{Forum}

The RCN Tuberculosis Visitors Forum is holding its annual conference in London on 18 October 1995 . Topics include legal aspects of nursing/accountability, compliance with tuberculosis treatment, medications and their interaction with TB drugs, 1993 National Survey of Notification of TB in England and Wales. RCN members $£ 47$; Non-members 665. Application forms from Sandra Treadwell. Telephone 0171409 3333. Fax: 01713551379.

\section{Fibres, particles and the lung: new perspectives}

The British Association for Lung Research (BALR) Summer Meeting entitled "Fibres, particles and the lung: new perspectives" will take place at the Edinburgh Conference Centre, Heriott Watt University, Edinburgh on 11-12 September 1995. For further information contact Dr R Cullen, Institute of Occupational Medicine, 8 Roxburgh Place, Edinburgh EH8 9SU. Telephone: 0131447 8460. Fax: 01314472822. 